\title{
Association of Anthropometric Obesity Measures with Physical Fitness in Adolescents: A Cross-sectional Comparative Study
}

\section{Mohammed Nazrul Islam ${ }^{1 *}$, Ram Lochan Yadav², Mohd Rasheeduddin Imran $^{1}$ and Sayeeda Anjum ${ }^{3}$}

${ }^{1}$ Department of Physiology, College of Pharmacy, University of Hafr Al Batin, Kingdom of Saudi Arabia

${ }^{2}$ Department of Physiology, Chitwan Medical College, Bharatpur, Nepal

${ }^{3}$ Department of Anatomy, College of Pharmacy, University of Hafr Al Batin, Kingdom of Saudi Arabia

*Corresponding Author: Mohammed Nazrul Islam, Professor of Physiology, College of Pharmacy, University of Hafr Al Batin, Kingdom of Saudi Arabia.
Received: February 15, 2021

Published: March 05, 2021

(C) All rights are reserved by Mohammed Nazrul Islam., et al.

\begin{abstract}
Obesity is increasing in Saudi Arabia at an alarming rate being ranked $29^{\text {th }}$ on a 2007 list of the fattest countries. Childhood/ adolescent obesity is a risk factor for cardiovascular disease and metabolic syndrome (MS) in adulthood and thus affects morbidity and mortality. Obesity also affects the cardiorespiratory and overall physical condition. Therefore, this study was aimed to evaluate if early obesity in life has a prediction of worse cardiac function and their association with cardiorespiratory physical fitness. This cross-sectional study included 90 adolescents with matched sex and age 14(2) years were divided into three groups- 1) Normal weight (NW), 2) Overweight (OW) and 3) Obese (OB) based on the BMI (body mass index) percentile for age and sex. Their waist circumference, waist-height ratio (WHtR), resting pulse rate and blood pressure were measured by standardized laboratory techniques. The modified Harvard step test was used to assess the physical fitness index (PFI \%) rated as excellent, good, fair and poor based on score. The obesity, cardiovascular and physical fitness parameters were compared between groups using Mann Whitney U test and the association between obesity and physical fitness parameters were evaluated by Spearman correlation test using SPSS V.20. The cardiovascular parameters-the pulse rate (NW Vs OW: 80(9)-89.5(13)/min, p < 0.001; and NW Vs OB: 80(9) - 94(12)/min, $\mathrm{p}<0.001$ ), SBP (NW Vs OW:117(5) -120(9) mmHg, p=0.003; and NW Vs OB:117(5) -121(9) mmHg, p < 0.001) and DBP (NW Vs OW: $74.33 \pm 3.64-79.43 \pm 5.54 \mathrm{mmHg}, \mathrm{p}<0.001$; and NW Vs OB:74.33 $\pm 3.64-81.20 \pm 3.91 \mathrm{mmHg}, \mathrm{p}<0.001$ ) were statistically significant between NW Vs OW and NW Vs OB groups. The physical fitness index level is statistically significant among NW Vs OW [1(1)-2.5(1), p $<0.001$ ], NW Vs OB [1(1)-4(0), P < 0.001]. The obesity measures-BMI/BMI percentiles were inversely related to the physical fitness score (rho=-0.589, p < 0.001), WC (rho=-0.565, p < 0.001) and WHtR ((rho=-0.565, p < 0.001). This study revealed poor cardiovascular measures with increased resting heart rate and blood pressure and the inverse association between cardiovascular physical fitness and obesity measures in the adolescent.
\end{abstract}

Keywords: Adolescent Obesity; Physical Fitness Index; BMI Percentiles; Waist Circumference; Resting Heart Rate

\section{Introduction}

Obesity has grown worldwide to the level of epidemic proportions in both children and adults [1]. Obesity in Saudi Arabia is a growing health concern, with health officials stating that it is one of the leading causes of preventable deaths in Saudi Arabia. According to Forbes, Saudi Arabia ranks $29^{\text {th }}$ on a 2007 list of the fattest coun- 
tries with a percentage of $68.3 \%$ of its citizens being overweight (BMI>25) [2]. According to CDC, WHO and National Institutes of Health, the criteria for obesity in childhood and adolescents has been made as classifying the BMI at the $85^{\text {th }}$ to $95^{\text {th }}$ percentiles for age and sex to identify children or teens (adolescents) who are overweight, and a BMI greater than or equal to the $95^{\text {th }}$ percentile to identify children or teens who are obese [3].

Childhood obesity is increasing in Saudi Arabia at an alarming rate. Relevant studies reported the highest prevalence of overweight and obesity with increased risk factors among higher parental education, and lifestyle factors, which included increased consumption of calorie-dense food and a sedentary lifestyle [4]. It was formerly seen as a problem of the middle-aged and elderly, but in recent times, childhood obesity has reached epidemic proportions $[5,6]$. Childhood obesity is a risk factor for cardiovascular disease and metabolic syndrome (MS) in adulthood and thus affects morbidity and mortality in later life. Up to $80 \%$ of obese children continued to become obese and thus affect morbidity and mortality in adult life [7].

Obesity is strongly correlated with the development of risk factors for non-communicable disorders, including hypertension, insulin resistance, type 2 diabetes mellitus, hypercholesterolemia, coronary heart disease, strokes and certain cancers and metabolic syndrome. Childhood and adolescent obesity are not just a current problem, but a potential cause of future health-related problems, including metabolic diseases [8]. Therefore, the cardiovascular risk factors are elevated in overweight with further abnormalities in those with obesity suggesting that even modest degrees of excess adiposity contribute to cardiovascular risk [9].

Recently, reduced physical activities were considered as one of the primary causes of childhood obesity [10]. Physical fitness is defined as "the ability to carry out daily tasks without fatigue". Healthrelated components include cardiorespiratory fitness (CRF), muscular endurance, muscular strength, and flexibility. Several studies have reported the negative association of physical fitness with childhood obesity [11,12]. Also, evidence from previous studies suggests that low CRF is a major risk factor of metabolic abnormalities, and is also known as an independent and strong predictor of metabolic syndrome [12].

The obesity and "super obesity" in adults could affect cardiac metabolic risk factors and performance [13]. However, the real impact of obesity especially at an early age on cardiac function is still largely unknown. Moreover, such changes in cardiac function, if any, in adolescent obesity and their association with cardiorespiratory physical fitness has remained to be observed in this region. Therefore, the aim of this study was to evaluate if early obesity in life has a worse cardiac function and their association with cardiorespiratory physical fitness.

\section{Materials and Methods}

\section{Subjects}

This cross-sectional study was conducted on adolescents aged between 11 and 15 years of both sexes recruited from elementary and middle schools. The study was conducted according to the guidelines of the Declaration of Helsinki and approval was taken by the institutional ethics review committee.

\section{The inclusion criteria}

- Both the parent or guardian and children give their written informed consent.

- The participant had to participate in physical activity test.

- Having $\geq 5$ th percentile of body mass index (BMI), according to the age- and gender-specific reference values of the 2007 CDC, WHO.

\section{Exclusion criteria}

- The subjects having below $5^{\text {th }}$ percentile of BMI according to age and sex.

- The subjects having any diagnosed systemic disease and under medication.

\section{Variables studied}

\section{Obesity and metabolic parameters}

The subjects were classified into 3 groups: (1) normal weight (NW, 5th-85th percentile); (2) overweight (OW, 85th-94th percentile) and (3) obesity (OB, $\geq 95$ th percentile) according to the age- and gender-specific reference values of 2007 WHO, CDC National Growth charts $[3,14]$. According to International Diabetes Federation criteria, adolescents with abdominal obesity $\left(\mathrm{WC} \geq 90^{\text {th }}\right.$ percentile for age and sex), Waist-height ratio $>0.4$ and 2 or more of the following criteria: $\mathrm{TG} \geq 150 \mathrm{mg} / \mathrm{dL} ; \mathrm{HDL} \leq 40 \mathrm{mg} / \mathrm{dL}$; fasting glucose $\geq 100 \mathrm{mg} / \mathrm{dL}$; and systolic $\mathrm{BP} \geq 130 \mathrm{mmHg}$ or diastolic $\mathrm{BP} \geq 85 \mathrm{mmHg}$ were diagnosed as Metabolic Syndrome related to obesity [15].

BP was measured twice using Dynosure Doctor Dt ${ }^{\circledR}$ mercury sphygmomanometers with an appropriate-sized cuff, and the av- 
erage of the two readings was taken as the final reading [16]. The waist circumference (WC) was measured midway between the tenth rib and the top of the iliac crest to the nearest $0.1 \mathrm{~cm}$, using a non-stretchable tape measure [17]. Weight was measured to the nearest $0.1 \mathrm{~kg}$ with a METTLER TOLEDO weighing machine, and height was measured to the nearest $0.1 \mathrm{~cm}$ using a wooden stadiometer. Weight and height were measured with the respondents wearing light school uniforms, without head or footwear, and without accessories such as purses, keys and mobile phones, to avoid overestimation [17]. BMI was calculated using the formula: weight $(\mathrm{kg}) /$ height squared $(\mathrm{m} 2)$. The calculated BMI was used to classify respondents as normal weight, overweight or obese, using the IOTF age- and sex-specific cut-off points [3,14].

\section{Physical fitness measurement and parameters}

Physical fitness was assessed using the physical activity fitness test- The modified Harvard step test [18]. Each participant was asked to place one foot on a 10-inch-high stool, step up, place both feet on the platform, straighten the legs and back, and immediately step down again, bringing down the same foot he/she first raised. This stepping up and down was continued at the rate of 20 steps per minute, following the rhythm of a metronome, for 3 minutes. Immediately after exercise, the participants were allowed to sit quietly on a chair and the heart rate (pulse rate) was measured for 30 seconds after exactly one minute and at the following time points: 1 to 1.5 minutes, 2 to 2.5 minutes and 3 to 3.5 minutes after the end of the exercise. Then physical fitness index (PFI) was calculated using the following equation.

Calculation of Physical fitness Index (\%) (PFI \%)

PFI = Duration of exercise in seconds x 100/2(pulse $1+2+3$ )

The cardiorespiratory physical fitness interpreted based on comparing the obtained score with the reference value given in the table below.

\begin{tabular}{|l|c|c|}
\hline \multirow{2}{*}{ PFI Rating } & \multicolumn{2}{|c|}{ Physical Fitness Index (PFI) } \\
\cline { 2 - 3 } & Male & Female \\
\hline Excellent & $>115$ & $>91$ \\
\hline Good & $103-115$ & $84-91$ \\
\hline Fair & $91-102$ & $77-83$ \\
\hline Poor & $<91$ & $<77$ \\
\hline
\end{tabular}

\section{Statistical analysis}

The data obtained were analyzed with SPSS (version 20; IBM Corporation, Armonk, NY, USA). The Shapiro-Wilk normality test was applied for all the descriptive parameters (Table 1). The comparison of the anthropometric, obesity and physical parameters between the groups were done by Mann Whitney U test for the non-parametric data and student t-test for the parametric data. Most of the data were non-parametric hence the Spearman's correlation test was applied to see the relation between obesity and physical fitness parameters. Statistical significance was considered to be $\mathrm{p} \leq 0.05$.

\section{Results}

The total 90 participants with median age -14(2) years, height - 158(8) cm and weight-60.44 $\pm 7.71 \mathrm{~kg}$ were divided into three groups namely; Normal weight (NW), Overweight (OW) and Obese (OB) based on the BMI percentile for age and sex. The participant's overall descriptive parameters with Shapiro-Wilk normality test were presented in Table 1. The age and sex of the participants were matched between the groups having 15 male and 15 female participants in each group. The age (Normal weight Vs Overweight: 13.5(3)- 14(4) years, $\mathrm{p}=0.45$; Normal weight Vs Obese: $13.5(3)$ - 13.5(2) years, $p=0.85$; and overweight Vs obese: 14(4) - 13.5(2) years, $p=0.46$ ) was comparable between the groups (Table 2, 3 and 4).

\begin{tabular}{|l|c|c|c|}
\hline Parameters & $\begin{array}{c}\text { Mean } \mathbf{\text { SD} /} \\
\text { Median } \\
\text { (Interquartile } \\
\text { Range) }\end{array}$ & $\begin{array}{c}\text { Confidence } \\
\text { interval CI } \\
\text { (95\%) }\end{array}$ & $\begin{array}{c}\text { Shapiro-Wilk } \\
\text { test } \\
\text { P-value }\end{array}$ \\
\hline Age (yrs.) & $14(2)$ & $13.13-13.67$ & $<0.001^{*}$ \\
\hline Ht. (cm) & $158(8)$ & $\begin{array}{c}157.73- \\
159.74\end{array}$ & $<0.001$ \\
\hline Wt. (kg) & $60.44 \pm 7.71$ & $58.83-62.06$ & 0.07 \\
\hline BMI (kg/m²) & $24.1(5.1)$ & $23.4-24.55$ & 0.002 \\
\hline $\begin{array}{l}\text { BMI } \\
\text { percentile }\end{array}$ & $91(15)$ & $84.71-89.11$ & $<0.001$ \\
\hline WC (cm) & $78(21)$ & $75.02-80.11$ & $<0.001$ \\
\hline WHtR & $0.49(0.13)$ & $0.47-0.5$ & 0.002 \\
\hline PR (per min) & $87.63 \pm 9.66$ & $85.61-89.66$ & 0.06 \\
\hline SBP (mmHg) & $120(8)$ & $118.20-$ & 0.01 \\
\hline DBP & $78.32 \pm 5.28$ & $77.22-79.43$ & 0.07 \\
\hline PFI & $3(2)$ & $2.37-2.83$ & $<0.001$ \\
\hline
\end{tabular}

Table 1: Participants $(\mathrm{N}=90)$ overall descriptive parameters with Shapiro-Wilk normality test. ${ }^{*} \mathrm{p} \leq 0.05$ considered as statistically significant. 
The anthropometric obesity measures- weight, BMI, BMI percentile for age and sex, waist circumference and waist-height ratio were statistically significant between the groups (Table 2, 3 and 4). The cardiovascular parameters including the pulse rate (NW Vs OW: 80(9)-89.5(13)/min, p < 0.001; and NW Vs OB: 80(9) 94(12)/min, p < 0.001), SBP (NW Vs OW:117(5) -120(9) mmHg, $\mathrm{p}=0.003$; and NW Vs OB:117(5) -121(9) mmHg, $\mathrm{p}<0.001$ ) and DBP (NW Vs OW: $74.33 \pm 3.64-79.43 \pm 5.54 \mathrm{mmHg}, \mathrm{p}<0.001$; and NW Vs OB:74.33 $\pm 3.64-81.20 \pm 3.91 \mathrm{mmHg}, \mathrm{p}<0.001$ ) were statistically significant between NW Vs OW and NW Vs OB groups but these parameters were not comparable between OW and OB group (Table 2, 3 and 4).

The Physical fitness index level is statistically significant among NW Vs OW [1(1)-2.5(1), p < 0.001], NW Vs OB [1(1)-4(0), P < 0.001] and OW Vs OB [2.5(1)-4(0), p < 0.001] groups. All the obesity parameters are positively correlated with cardiorespiratory variables and their correlation were statistically significant (Table 5). Likewise, the obesity parameters revealed a strong negative correlation with the physical fitness score and a positive correlation with the PFI grade from excellent to poor (Table 5). Therefore, this finding revealed that the physical fitness deteriorates and shifts from excellence to poor category on increasing obesity measures.

\begin{tabular}{|l|c|c|c|}
\hline Parameters & $\begin{array}{c}\text { Normal weight } \\
\text { Median } \\
\text { (Interquartile } \\
\text { Range)/Mean } \\
\mathbf{\pm} \text { SD }\end{array}$ & $\begin{array}{c}\text { Overweight } \\
\text { Median } \\
\text { (Interquartile } \\
\text { Range)/Mean } \\
\mathbf{\pm S D}\end{array}$ & $\begin{array}{c}\text { Mann- } \\
\text { Whitney U } \\
\text { test/student } \\
\text { t test } \\
\text { P-value }\end{array}$ \\
\hline -Age (yrs.) & $13.5(3)$ & $14(4)$ & 0.45 \\
\hline Ht. (cm) & $158(8)$ & $157(7)$ & 0.69 \\
\hline Wt. (Kg) & $52.67 \pm 4.27$ & $61 \pm 4.29$ & $<0.001$ \\
\hline BMI & $20.8(1.45)$ & $24.2(2.15)$ & $<0.001$ \\
\hline BMI \\
percentile & $77.5(14)$ & $91(5)$ & $<0.001$ \\
\hline WC & $64(4)$ & $78(6)$ & $<0.001$ \\
\hline WHtR & $0.4(0.03)$ & $0.49(0.04)$ & $<0.001$ \\
\hline PR & $80(9)$ & $89.5(13)$ & $<0.001$ \\
\hline SBP & $117(5)$ & $120(9)$ & 0.003 \\
\hline DBP & $74.33 \pm 3.64$ & $79.43 \pm 5.54$ & $<0.001$ \\
\hline PFI & $1(1)$ & $2.5(1)$ & $<0.001$ \\
\hline
\end{tabular}

Table 2: Comparison of anthropometric parameters between normal weight $(n=30)$ and overweight $(n=30)$ adolescents.

\begin{tabular}{|l|c|c|c|}
\hline Parameters & $\begin{array}{c}\text { Normal } \\
\text { weight } \\
\text { Median } \\
\text { (Interquartile } \\
\text { Range)/Mean } \\
\pm \text { SD }\end{array}$ & $\begin{array}{c}\text { Obese } \\
\text { Median } \\
\text { (Interquartile } \\
\text { Range)/Mean } \\
\pm \text { SD }\end{array}$ & $\begin{array}{c}\text { Mann-Whitney } \\
\text { U test/ Student } \\
\text { t-test } \\
\text { P-value }\end{array}$ \\
\hline Age (yrs.) & $13.5(3)$ & $13.5(2)$ & 0.85 \\
\hline Ht. (cm) & $158(8)$ & $157.5(10)$ & 0.98 \\
\hline Wt. (Kg) & $52.67 \pm 4.27$ & $67.67 \pm 5.4$ & $<0.001$ \\
\hline BMI & $20.8(1.45)$ & $26.8(2.05)$ & $<0.001$ \\
\hline BMI percentile & $77.5(14)$ & $96(2)$ & $<0.001$ \\
\hline WC & $64(4)$ & $90(12)$ & $<0.001$ \\
\hline WHtR & $0.4(0.03)$ & $0.57(0.05)$ & $<0.001$ \\
\hline PR & $80(9)$ & $94(12)$ & $<0.001$ \\
\hline SBP & $117(5)$ & $121(9)$ & $<0.001$ \\
\hline DBP & $74.33 \pm 3.64$ & $81.20 \pm 3.91$ & $<0.001$ \\
\hline PFI & $1(1)$ & $4(0)$ & $<0.001$ \\
\hline
\end{tabular}

Table 3: Comparison of anthropometric parameters between normal weight $(n=30)$ and obese $(n=30)$ adolescents.

\begin{tabular}{|l|c|c|c|}
\hline Parameters & $\begin{array}{c}\text { Overweight } \\
\text { Median } \\
\text { (Interquartile } \\
\text { Range)/Mean } \\
\pm \text { SD }\end{array}$ & $\begin{array}{c}\text { Obese } \\
\text { Median } \\
\text { (Interquartile } \\
\text { Range)/Mean } \\
\mathbf{\pm S D}\end{array}$ & $\begin{array}{c}\text { Mann-Whitney } \\
\text { U test/student } \\
\text { t-test } \\
\text { P-value }\end{array}$ \\
\hline Age (yrs.) & $14(4)$ & $13.5(2)$ & 0.46 \\
\hline Ht. (cm) & $157(7)$ & $157.5(10)$ & 0.78 \\
\hline Wt. (Kg) & $61 \pm 4.29$ & $67.67 \pm 5.4$ & $<0.001$ \\
\hline BMI & $24.2(2.15)$ & $26.8(2.05)$ & $<0.001$ \\
\hline BMI percentile & $91(5)$ & $96(2)$ & $<0.001$ \\
\hline WC & $78(6)$ & $90(12)$ & $<0.001$ \\
\hline WHtR & $0.49(0.04)$ & $0.57(0.05)$ & $<0.001$ \\
\hline PR & $89.5(13)$ & $94(12)$ & 0.05 \\
\hline SBP & $120(9)$ & $121(9)$ & 0.39 \\
\hline DBP & $79.43 \pm 5.54$ & $81.20 \pm 3.91$ & 0.15 \\
\hline PFI & $2.5(1)$ & $4(0)$ & $<0.001$ \\
\hline
\end{tabular}

Table 4: Comparison of anthropometric parameters between overweight $(n=30)$ and obese $(n=30)$ adolescents. 


\begin{tabular}{|l|c|c|c|c|c|c|}
\hline \multicolumn{2}{|c|}{ Variables } & PR & SBP & DBP & PFI & PFI-G \\
\hline Wt. & rho & 0.477 & 0.308 & 0.520 & -0.537 & 0.670 \\
& P value & $<0.001$ & 0.003 & $<0.001$ & $<0.001$ & $<0.001$ \\
\hline BMI & rho & 0.495 & 0.281 & 0.551 & -0.624 & 0.732 \\
& P value & $<0.001$ & 0.003 & $<0.001$ & $<0.001$ & $<0.001$ \\
\hline BMI & rho & 0.568 & 0.370 & 0.593 & -0.589 & 0.827 \\
percentile & P value & $<0.001$ & $<0.001$ & $<0.001$ & $<0.001$ & $<0.001$ \\
\hline WC & rho & 0.599 & 0.383 & 0.541 & -0.565 & 0.769 \\
& P value & $<0.001$ & $<0.001$ & $<0.001$ & $<0.001$ & $<0.001$ \\
\hline WHtR & rho & 0.599 & 0.355 & 0.519 & -0.565 & 0.779 \\
& P value & $<0.001$ & 0.001 & $<0.001$ & $<0.001$ & $<0.001$ \\
\hline
\end{tabular}

Table 5: Spearman's correlation of obesity parameters with cardiorespiratory and physical fitness variables $(\mathrm{N}=90)$.

\section{Discussion}

We explored the difference and association between anthropometric obesity measures with cardiorespiratory physical fitness index determined by modified Harvard step stool test among normal weight (NW), overweight (OW) and obese (OB) adolescents. This cross-sectional comparative study revealed that the anthropometric obesity measures- weight, BMI, BMI percentile for age and sex, waist circumference and waist-height ratio were statistically significant among three groups. The cardiovascular parameters including pulse rate, SBP and DBP were also significantly elevated in OW and OB groups than the NW adolescents (Table 2, 3 and 4). This could be due to altered cardiovascular autonomic activity in overweight and obese adolescents thereby increasing the resting heart rate and arterial blood pressure. These findings are in line with the study by Baba R., et al. reporting such hemodynamic abnormalities with adolescent obesity explained by impaired autonomic nerve function [19]. Similar to our findings, other studies reported the relationship between elevated resting heart rate and increased systolic and diastolic blood pressure in both sexes and pulse pressure even after controlling for potential confounders, such as general and abdominal obesity [20,21].

In our study, waist circumference (WC) and waist-height ratio (WHtR) were significantly higher in overweight and obese adolescents than the normal weight adolescents. This study also highlighted the significance of WC and WHtR in defining the obesity which could have altered the resting heart rate (RHR) and blood pressure among them irrespective of BMI percentile and amount of distribution of fat. Similar findings were reported in previous studies mentioning that BP was positively associated with RHR with high WCs, highlighting the positive association between sympathetic activity and BP in children regardless of the distribution and the amount of central fat $[22,23]$.

After analyzing correlation, this study revealed a significant positive association of individual anthropometric obesity measures with the cardiovascular physical parameters. As the BMI/BMI percentile for age and sex of adolescent's progress across the NW, OW and $\mathrm{OB}$ groups, the resting heart rate or pulse rate $(\mathrm{rho}=+0.495$, $\mathrm{p}<0.001$ ), SBP (rho=+0.281, p=0.003) and DBP (rho=+0.551, p < 0.001 ) significantly elevated. Such positive correlations were also true for the other obesity markers including WC and WHtR (Table 5). Our findings are incoherence with the previous reports stating the adolescents classified with high body mass index, waist circumference, and waist to height ratio demonstrated a high association of presenting high blood pressure [24]. Our study also highlighted the importance of WC and WHtR including BMI percentile for age and sex. The anthropometric variables studied showed a moderate predictive capacity for HBP, highlighting WC, which showed the strongest association with high blood pressure in the infant and child population [25].

Expectedly, our study revealed that the physical fitness score significantly decreased on moving across NW to OW and OW to OB group adolescents. There are five components in physical fitness including muscular strength, cardiovascular endurance, muscular endurance, flexibility and body fat composition [26]. All these components are relative to each other. In the above discussion, we have pointed that OW and OB group adolescents had poorer cardiovascular parameters compared to normal or healthy weight group. These poor cardiovascular parameters along with reduced physical activity could be the reason for reduced physical fitness scores among the OW and OB adolescents. These findings are supported by previous reports $[27,28]$.

The physical fitness of thin and normal youth was, with few exceptions, significantly better than the physical fitness of overweight and obese youth in each age group. In contrast to our study, physical fitness performances did not consistently differ, on average, between thin and normal weight and between overweight and obese youths [29]. However, in our study, there was a significantly low physical fitness score in obese than both the OW and NW adolescents. 
Moreover, the individual obesity measures were inversely related to the physical fitness score and PFI grading proceeded from excellence fitness to poor fitness on increasing BMI/BMI percentiles for age and sex (rho=-0.589, p < 0.001), WC (rho=-0.565, p < 0.001) and WHtR ( $(r h o=-0.565, \mathrm{p}<0.001)$. This shows the physiological importance of establishing the relationship between the body fat or obesity measures to physical activity or fitness. By combining these findings with the poorer cardiovascular measures in OW and $\mathrm{OB}$ adolescents, we can conclude the poor cardiovascular and physical fitness among $\mathrm{OW} / \mathrm{OB}$ adolescents. These findings with an inverse relationship of obesity measures and physical fitness are consistent with the previous report [30].

\section{Conclusion}

The findings of the present study support previous findings showing poor cardiovascular measures with increased resting heart rate and arterial blood pressure and the inverse association between cardiovascular-physical fitness and obesity measures in the adolescent. Further studies are needed to establish the relationship between cardiorespiratory physical fitness and obesity across different ages, genders and regions.

\section{Conflict of Interest}

We declare that we do not have any conflict of interest.

\section{Acknowledgments}

This study was funded by a research grant (G-116-2020) from the University of Hafr Al Batin, Ministry of Education, Kingdom of Saudi Arabia. The authors would like to thank the administration and staff of the schools who participated in the study, along with the school children and their parents. The assistance provided by the team members is also gratefully acknowledged.

\section{Bibliography}

1. Casazza K., et al. "Presumptions and facts about obesity". The New England Journal of Medicine 368 (2013): 446-454.

2. Lauren S. "World's Fattest Countries". Forbes. February 8 (2007).

3. Barlow SE and Expert Committee. "Expert committee recommendations regarding the prevention, assessment, and treatment of child and adolescent overweight and obesity: summary report". Pediatrics 120.4 (2007): S164-192.
4. Hammad SS and Berry DC. "The Child Obesity Epidemic in Saudi Arabia: A Review of the Literature". Journal of Transcultural Nursing 28.5 (2017): 505-515.

5. Nader PR., et al. "Identifying risk for obesity in early childhood". Pediatrics 118 (2006): e594-e601.

6. Kelishadi R. "Childhood overweight, obesity, and the metabolic syndrome in developing countries". Epidemiology Review 29 (2007): 62-76.

7. Stewart L. "Childhood obesity". Medicine 39 (2011): 42-44.

8. Lavie CJ., et al. "Obesity and cardiovascular disease: risk factor, paradox and impact of weight loss". Journal of the American College of Cardiology - JACC 53 (2009):1925-1932.

9. Zhu HYan., et al. "Relationships of cardiovascular phenotypes with a healthy weight, at risk of overweight, and overweight in US youths". Pediatrics 121.1 (2008): 115- 122.

10. Deforche B., et al. "Physical fitness and physical activity in obese and nonobese Flemish youth". Obesity Research 11 (2003): 434-441.

11. Minck MR., et al. "Physical fitness, body fatness, and physical activity: The Amsterdam Growth and Health Study". American Journal of Human Biology 12 (2000): 593-599.

12. Kumagai S., et al. "Relative contributions of cardiorespiratory fitness and visceral fat to metabolic syndrome in patients with diabetes mellitus". Metabolic Syndrome and Related Disorders 3 (2005): 213-220.

13. Rider OJ., et al. "Structural and Metabolic Effects of Obesity on the Myocardium and the Aorta". Obesity Facts 7 (2014): 329338.

14. Speiser PW., et al. "Childhood obesity". The Journal of Clinical Endocrinology and Metabolism 90 (2005): 1871-1887.

15. Zimmet P., et al. "The metabolic syndrome in children and adolescents: an IDF consensus report". Pediatric and Diabetes 8 (2007): 299-306.

16. Taksande A., et al. "Distribution of blood pressure in schoolgoing children in rural area of Wardha district, Maharashtra, India”. Annals of Pediatric Cardiology 1.2 (2008): 101-106. 
17. McCarthy HD., et al. "Central overweight and obesity in British youth aged 11-16 years: cross-sectional surveys of waist circumference". BMJ 326.7390 (2003): 624-627.

18. Gallagher J and Brouha L. "A simple method of testing the physical fitness of boys". Yale Journal of Biology and Medicine 15 (1943): 667-677.

19. Baba R., et al. "Adolescent obesity adversely affects blood pressure and resting heart rate". Circulation Journal 71.5 (2007): 722-726.

20. Christofaro DGD., et al. "Relationship between Resting Heart Rate, Blood Pressure and Pulse Pressure in Adolescents". Arquivos Brasileiros de Cardiologia 108.5 (2017): 405-410.

21. Hu J., et al. "Association of elevated resting pulse rate with increased risk of hypertension development in children: A prospective study in Suzhou, China". Medicine (Baltimore) 96.32 (2017): e7696.

22. Dong B., et al. "The association between resting heart rate and blood pressure among children and adolescents with different waist circumferences". European Journal of Pediatrics 174.2 (2015): 191-197.

23. Silva DAS., et al. "Association between resting heart rate and health-related physical fitness in Brazilian adolescents". BioMed Research International 28 (2018): 3812197.

24. Farah BQ., et al. "Association between resting heart rate and cardiovascular risk factors in adolescents". European Journal of Pediatrics 174.12 (2015): 1621-1628.

25. Vaquero-Álvarez M., et al. "Diagnostic precision of anthropometric variables for the detection of hypertension in children and adolescents". International Journal of Environmental Research and Public Health 17.12 (2020): 4415.

26. Daniels RS. "From critical periods for abnormal weight gain in children and adolescents. Handbook of Pediatric Obesity: Etiology, Pathophysiology and Prevention". Edi: Goran SMI, Sothern MS. Taylor and Francis Group, Florida (2006): 67-75.

27. Minder CM., et al. "Relation between self-reported physical activity level, fitness, and cardiometabolic risk". American Journal of Cardiology 113.4 (2014): 637-643.
28. Chen JL., et al. "Correlates of physical fitness and activity in Taiwanese children". International Nursing Review 55 (2008): 81-88.

29. Lopes VP., et al. "Body mass index and physical fitness in Brazilian adolescents". Journal of Pediatrics 95.3 (2019): 358-365.

30. Kim HJ., et al. "Relationships of physical fitness and obesity with metabolic risk factors in children and adolescents: Chungju city cohort study". Annals of Pediatric Endocrinology and Metabolism 21.1 (2016): 31-38.

\section{Volume 5 Issue 4 April 2021 \\ (C) All rights are reserved by Mohammed Nazrul Islam., et al.}

